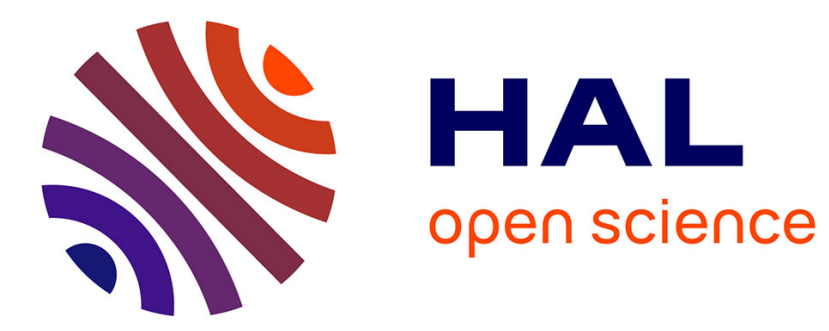

\title{
Spécificités de la prise en charge de la femme épileptique
} Sophie Dupont

\section{To cite this version:}

Sophie Dupont. Spécificités de la prise en charge de la femme épileptique. La Presse Médicale, 2018, 47 (3), pp.251 - 260. 10.1016/j.lpm.2018.01.020 . hal-01827503

\section{HAL Id: hal-01827503 https://hal.sorbonne-universite.fr/hal-01827503}

Submitted on 2 Jul 2018

HAL is a multi-disciplinary open access archive for the deposit and dissemination of scientific research documents, whether they are published or not. The documents may come from teaching and research institutions in France or abroad, or from public or private research centers.
L'archive ouverte pluridisciplinaire HAL, est destinée au dépôt et à la diffusion de documents scientifiques de niveau recherche, publiés ou non, émanant des établissements d'enseignement et de recherche français ou étrangers, des laboratoires publics ou privés. 
Spécificités de la prise en charge de la femme épileptique

Epilepsie cataméniale

Contraception

Grossesse

Ménopause

${ }^{1,2,3,4}$ Sophie Dupont

${ }^{1}$ Epilepsy Unit, Hôpital de la Pitié-Salpêtrière, APHP, Paris, France

${ }^{2}$ Rehabilitation Unit, Hôpital de la Pitié-Salpêtrière, APHP, Paris, France

${ }^{3}$ Centre de Recherche de l'Institut du Cerveau et de la Moëlle Epinière (ICM), UMPC-UMR

7225 CNRS-UMRS 975 INSERM, Paris, France

${ }^{4}$ Université Pierre et Marie Curie, Paris 6, France

\section{Correspondance:}

Pr S Dupont, Unité d'Epilepsie

Hôpital de la Salpêtrière

47, boulevard de l'Hôpital

75651 Paris cedex 13, France

Phone: (33) 142164115

FAX: (33) 142160303

E-mail : sophie.dupont@psl.aphp.fr

Déclaration des liens d'intérêt : l'auteur déclare avoir reçu des honoraires en tant que consultante ou oratrice des laboratoires EISAI, UCB et GSK. 


\section{Points essentiels}

1. L'épilepsie cataméniale, définie comme l'exacerbation de la fréquence des crises dans une phase déterminée du cycle menstruel, toucherait $35 \%$ des femmes.

2. Chez les femmes présentant une épilepsie cataméniale avec recrudescence périmenstruelle des crises, un traitement par progestérone peut s'avérer efficace.

3. En cas de prise de MAE inducteur enzymatique, la contraception hormonale est déconseillée (pilule estroprogestative ou progestative, implant progestatif, patchs ou anneaux hormonaux).

4. Le valproate de sodium, du fait de son potentiel tératogène malformatif élevé, de ses possibles effets cognitifs délètères et du risque autistique n'est pas indiqué pendant la grossesse.

5. A ce jour, les MAE les plus recommandés pour la grossesse sont la Lamotrigine et le Lévétiracétam.

6. Un monitoring des taux plasmatiques de médicaments est recommandé pendant la grossesse.

7. La décision d'allaitement demeure individuelle en fonction essentiellement du terme de l'accouchement et du type de traitement antiépileptique.

\section{Key points}

Specific aspects of the management of women with epilepsy

Catamenial epilepsy/Contraception/Pregnancy/Menopause

1. Catamenial epilepsy, defined as the exacerbation of the frequency of seizures in a given phase of the menstrual cycle, affects $35 \%$ of women.

2. In women with catamenial epilepsy with perimenstrual seizures, progesterone therapy may be effective.

3. In case of enzyme inducer AEDs, hormonal contraception is deprecated (estroprogestative or progestative pill, progestative implant, patches or hormonal rings).

4. Because of its high malformative teratogenic potential, its possible depressive cognitive effects and the autistic risk, sodium valproate is not indicated during pregnancy.

5. To date, the most recommended AEDs for pregnancy are Lamotrigine and Levetiracetam.

6. Plasma drug monitoring is recommended during pregnancy.

7. The decision to breastfeed remains individual depending mainly on the term of delivery and the type of antiepileptic treatment. 
On dénombre actuellement 300000 patientes épileptiques en France. Ces patientes devront faire face toute leur vie à des aspects spécifiques de leur maladie liés tout à la fois aux effets des crises et des médicaments anti-épileptiques (MAE) sur leur fonction hormonale, leur sexualité ou leur désir de reproduction. Parce que la prise en charge d'une patiente épileptique diffère clairement de celle d'un patient épileptique, différents aspects très spécifiques à la femme épileptiques seront abordés dans cette mise au point : épilepsie cataméniale, contraception, grossesse, ménopause.

\section{Epilepsie cataméniale}

L'épilepsie cataméniale est définie comme l'exacerbation de la fréquence des crises dans une phase déterminée du cycle menstruel [1]. L’épilepsie cataméniale peut se voir dans tout type d'épilepsie, généralisée ou focale, toucherait $35 \%$ des femmes et serait fréquemment associée à une pharmacorésistance. Pour rappel, le cycle menstruel est composé d'une phase folliculaire et d'une phase lutéale rythmées par l'ovulation et les menstruations. Ces deux événements sont marqués par des modifications hormonales spécifiques: une poussée préovulatoire des taux d'estradiol et une chute prémenstruelle des taux de progestérone [2]. Herzog, le neurologue américain spécialiste de l'épilepsie cataméniale a décrit trois principaux patterns d'épilepsie cataméniale :

- Le pattern $\mathrm{C} 1$ : périmenstruel, exacerbation des crises sur une période de 3 jours avant/après la survenue des menstruations, représenterait 35,7\% à 40\% [3] des épilepsies cataméniales

- Le pattern C2 : périovulatoire, exacerbation des crises sur une période de 5 jours autour de l'ovulation, représenterait $28,5 \%$ à $34 \%$ [3] des épilepsies cataméniales

- Le pattern C3 : anovulatoire, exacerbation des crises sur toute la deuxième période lutéale du cycle, représenterait 41,4\% à 47,4\% [3] des épilepsies cataméniales

Les patterns C1et C2 (cycles ovulatoires) peuvent coexister chez une même patiente. Sur un plan physiopathologique, l'épilepsie cataméniale est principalement expliquée par les propriétés neuroactives des hormones sexuelles (schématiquement effet proconvulsivant de l'estradiol et anticonvulsivant de la progestérone), et par les variations de ces hormones au cours du cycle menstruel. Les crises périmenstruelles (C1) seraient ainsi liées à l'effondrement prémenstruel de la progestérone s'accompagnant d'un effondrement de l'allopregnanolone, modulateur allostérique positif des récepteurs GABAA et de modifications dans la composition des sous unités du récepteur GABAA, notamment la sous unité $\alpha 4$ insensible aux benzodiazépines et au GABA. Les crises périovulatoires (C2) seraient liées au pic d'estradiol et 
les crises anovulatoires (C3) à un taux bas constant de progestérone en deuxième phase du cycle. Dans tous les cas, il paraît donc séduisant de proposer des traitements renforçant les taux circulants de progestérone pour rétablir un ratio estradiol/progestérone protecteur. Une étude randomisée, contre placebo, en double aveugle de l'efficacité de la progestérone (200 mg 3 fois/jour du $14^{\mathrm{e}}$ au $28^{\mathrm{e}}$ jour du cycle) a ainsi été conduite dans le traitement des épilepsies cataméniales focales pharmacorésistantes [4] et n'a pas retrouvé globalement d'efficacité du traitement progestatif. Néanmoins, une analyse post-hoc [3] a démontré une efficacité de la progestérone dans le type $\mathrm{C} 1$ d'épilepsie cataméniale avec un taux de répondeurs passant de $21,3 \%$ tout type d'épilepsies cataméniales confondu à $57,1 \%$ pour les seuls $\mathrm{C} 1$ (versus respectivement $19,6 \%$ et $20 \%$ sous placebo). Il semble donc raisonnable à ce jour de proposer un traitement par progestérone aux patientes souffrant d'épilepsie cataméniale avec recrudescence périmenstruelles des crises (type $\mathrm{C} 1$ ).

\section{Contraception}

La contraception, droit absolu des femmes, s'avère encore plus cruciale chez la femme épileptique chez qui la grossesse doit absolument se programmer. Une étude récente auprès des patientes épileptiques Américaines a montré que $65 \%$ de celles qui étaient traités par un MAE inducteur enzymatique ignoraient que leur traitement antiépileptique réduisait l'efficacité de leur contraception [5]. Pire, une grossesse non programmée sur quatre serait liée à l'inefficacité de la méthode contraceptive prescrite chez les patientes épileptiques Américaines [6]. Il paraît donc urgent qu'une information éclairée et concertée des gynécologues et des neurologues puisse être apportée aux patientes épileptiques en désir de contraception.

Les recommandations de l'OMS en la matière sont claires [7] (tableau 1) :

- le dispositif intra utérin (DIU) (hormonal ou au cuivre) est la méthode contraceptive de choix pour les patientes épileptiques quel que soit leur traitement

- chez des patientes traitées par des MAE inducteurs enzymatiques, on déconseille : les pilules estroprogestatives (quelque soit le dosage ou la génération) ou progestative pures (quelque soit le dosage), les implants progestatifs [8], les patchs ou anneaux vaginaux. Les méthodes contraceptives suivantes sont alors recommandées: DIU au levonorgestrel ou au cuivre, méthodes barrières, acétate de medroxyprogesterone injectable (DMPA) 
- chez des patientes traitées par des MAE inducteurs enzymatiques, il n'est pas établi que la pilule du lendemain soit efficace (même en doublant la dose), on préconise alors plutôt le DIU d'urgence

- chez des patientes traitées par des MAE non inducteurs enzymatiques, la contraception orale estroprogestative ou progestative est possible, avec trois réserves :

- le Felbamate (Taloxa), habituellement inhibiteur enzymatique, est inducteur du cytochrome CYP3A4 et diminue les taux circulants de gestodene (dérivé de la progestérone) [9], on recommande donc dans ce cas d'éviter la contraception orale, notamment progestative

- la Lamotrigine (Lamictal) ne diminue pas l'efficacité de la pilule estroprogestative mais celle-ci par un mécanisme de conjugaison glucuronide peut significativement abaisser les taux plasmatiques circulants de Lamotrigine [10]. Il convient donc de surveiller les taux plasmatiques de Lamotrigine et éventuellement d'en augmenter les doses

- le Valproate de sodium (Dépakine) est inhibiteur enzymatique et n'affecte donc pas la contraception hormonale. Une étude suggère que la contraception orale hormonale pourrait réduire les taux circulants de Valproate de sodium [11], incitant à la prudence.

Outre l'efficacité contraceptive, l'autre question posée par les patientes épileptiques est celle de l'impact d'une contraception hormonale sur l'équilibre de l'épilepsie. Une étude récente [12] a montré que $72 \%$ des patientes épileptiques ne mentionnaient pas de modification de la fréquence de leurs crises après instauration d'une contraception hormonale. A contrario, 28\% notaient une exacerbation de fréquence, ce qui reste à prendre en compte dans le choix ou l'ajustement de la méthode contraceptive.

\section{Grossesse}

\section{Programmation de la grossesse}

Dans tous les cas, un projet de grossesse demandera une programmation et une information concertée du couple avec une consultation préconceptionnelle. L'adaptation du traitement antiépileptique pourra alors être requise avant la mise en œuvre de la grossesse : arrêt du traitement, réduction du nombre et de la posologie des médicaments, remplacement d'un MAE très tératogène par un autre.

Le bénéfice réel de la prescription préconceptionnelle de folates à la dose de $5 \mathrm{mg} / \mathrm{j}$ (elle est admise à $0,4 \mathrm{mg} / \mathrm{j}$ une fois la grossesse déclarée dans la population générale) est actuellement très controversé [13]. Les folates continuent néanmoins à être initiés 2 à 3 mois avant le 
début de la grossesse, et poursuivis pendant au moins les 4 premiers mois de grossesse, en précisant bien aux patientes que cette prescription n'annule pas le risque de malformations, notamment d'absence de fermeture du tube neural (spina bifida).

\section{Grossesse}

\section{Risque tératogène}

Le risque malformatif tératogène lié à l'emploi des MAE est actuellement sous le feu de l'actualité avec le Valproate de sodium. Ce risque est réel mais dépend de trois paramètres : la molécule antiépileptique même, la dose, le nombre de MAE co-administrés.

\section{Données de monothérapie}

L'avènement des grands registres de grossesse américains [14, anglo-saxons [15,16] et européens (EURAP) [17] qui à ce jour ont colligé plus de 42000 patientes enceintes sous MAE a permis de démontrer qu'en monothérapie, tous les MAE n'ont pas le même potentiel tératogène. On note ainsi du plus tératogène au moins tératogène :

- Valproate de sodium : possède le plus fort potentiel tératogène en monothérapie, tout type de malformations confondu, ce risque tératogène est dose dépendant : $24 \%$ de malformations à un an au-delà d'une dose quotidienne de1500 mg/j et 5.6\% à un an en deçà de $750 \mathrm{mg} / \mathrm{j}$ [17]. Ce fort risque tératogène, allié à d'autres risques inhérents au médicament (neurodéveloppemental, trouble autistique, cf. chapitre infra) ont conduit les autorités Européennes et l'ANSM à demander une restriction d'utilisation du Valproate de Sodium chez la fillette et chez les femmes en âge de procréer. Depuis Juin 2015, la prescription de toutes les spécialités contenant du Valproate : Dépakine® (Valproate de sodium), Dépakote ${ }^{\circledR}$ (divalproate de sodium), Dépamide ${ }^{\circledR}$ (valpromide), Micropakine ${ }^{\circledR}$ (Valproate de sodium + acide valproïque) et génériques) est ainsi désormais strictement réglementée chez les patientes épileptiques : initiation réservée aux neurologues, psychiatres ou pédiatres, renouvellement nécessitant une prescription annuelle d'un de ces trois spécialistes, prescription annuelle subordonnée à la co-signature d'un accord de soins par le médecin spécialiste et par la patiente (ou son représentant légal), accord de soins signé requis pour la délivrance du médicament chez le pharmacien (fig. 1).

- Phénobarbital (Gardénal, Alepsal) : tératogénicité avérée, sur 709 grossesses recensées dans le revue Cochrane (23 études), on retrouve une prévalence de malformations congénitales majeures de 7,1\% sous phénobarbital. Avec là encore dans EURAP une claire dose dépendance (taux de malformations de 13,7\% au-delà de $150 \mathrm{mg} / \mathrm{j}$ ). La prise de phénobarbital est associée à un taux plus élevé de malformations cardiaques 
- Phénytoïne (Dihydan) : tératogénicité avérée, sur 1279 enfants (22 études), la prévalence de malformations congénitales majeures est de $5,38 \%$ corrigée à $6,26 \%$ pour la variance dans la revue Cochrane

- Topiramate (Epitomax) : tératogénicité avérée, sur 473 enfants exposés, le taux de malformations est de 4,28\%, principalement des hypospadias et des fentes labio-palatines [20], sans notion à ce jour de dose dépendance

- Carbamazépine (Tégretol) : potentielle tératogénicité dose dépendante : deux revues récentes dont une revue Cochrane [18,19] montrent que la carbamazépine peut être associée à une augmentation du risque tératogène (OR à 1,37, prévalence de $3,71 \%$ corrigée à 4,93\% pour la variance dans l'étude Cochrane). Cette augmentation semble très dose dépendante avec notamment un risque accru au-delà de $1000 \mathrm{mg} / \mathrm{j}(8,7 \%$ et 5,3\% respectivement dans les registres EURAP et anglais). Entre $400 \mathrm{mg} / \mathrm{j}$ et $1000 \mathrm{mg} / \mathrm{j}$, les données sont discordantes avec une absence d'augmentation du risque tératogène dans les registres Américain et Anglais et une augmentation du risque à 3,2\% dans le registre Européen, ce pour des cohortes d'enfants équivalentes Dans la revue Cochrane, la survenue de fentes labio-palatines était significativement associée à la prise de carbamazépine pendant la grossesse alors que le taux de spina bifida ou de malformations cardiaques était identique à celui de la population d'enfants contrôles nés de mères non épileptiques ou de mères épileptiques traitées.

- Lamotrigine (Lamictal) : absence de tératogénicité avérée à ce jour, sur 4195 enfants exposés, la prévalence de malformations est de 2,31\%. EURAP avait suggéré un éventuel effet dose-dépendant (taux de 3,6\% au-delà de $300 \mathrm{mg} / \mathrm{j}$ versus $1,7 \%$ en deçà de $300 \mathrm{mg} / \mathrm{j}$ ) non retrouvé dans les registres Anglais et Américain. Le risque accru de fentes labiopalatines sous Lamotrigine mis un temps en exergue dans le registre Américain a été infirmé depuis [21]

- Lévétiracetam (Keppra) : absence de tératogénicité avérée à ce jour, sur 817 enfants exposés, la prévalence de malformations est de 1,77\% sans notion à ce jour de dose dépendance Par ailleurs, on dispose de données parcellaires (rassurantes ou non) mais sur de trop faibles effectifs pour pouvoir conclure pour les MAE suivants :

- oxcarbazépine (Trileptal) : sur 238 enfants exposés, prévalence de malformations de 2,39\% [22]

- gabapentine (Neurontin) : sur 190 enfants exposés, prévalence de malformations de 1,47\% [18]

- pregabaline (Lyrica) : sur 125 enfants exposés, prévalence de malformations de 6,4\% [23] 
- zonisamide (Zonegran) : sur 90 enfants exposés, prévalence de malformations de 0,28\% [18]

Pour tous les autres MAE, l'absence de données plaide en faveur d'une abstention d'utilisation pendant la grossesse en principe de précaution.

\section{Données de polythérapie}

La polythérapie augmente le risque de malformations fœtales de manière significative, notamment en cas d'association avec le Valproate de sodium. Même lorsqu'on exclue les polythérapies comprenant du Valproate de sodium, Vadja et al. [24] ont montré qu'avec une polythérapie le taux de malformations fœtales restait plus élevé qu'avec une monothérapie $(6,94 \%$ versus $3,64 \%)$, notamment lorsque cette polythérapie incluait du Topiramate (taux de $14,94 \%$ de malformations).

\section{Devenir cognitif des enfants nés de mères épileptiques traitées par MAE}

En 2004, Adab et al. ont fait état d'un possible retard psychomoteur chez des enfants nés de mères ayant pris du Valproate pendant leur grossesse [25]. Ce retard concernait au départ essentiellement le QI verbal, mais dès 2009, Meador et al. ont démontré que le retard était plus global (QI plus bas de 9 points chez les enfants exposés au Valproate par rapport à ceux exposés à la lamotrigine) avec un effet possiblement dose-dépendant [26]. Ce retard, qui ne semble pas s'amender avec le temps, est également retrouvé chez des enfants plus âgés scolarisés [27]. Par ailleurs des difficultés comportementales (hyperactivité, troubles de concentration, maladresse motrice) ont également été rapportés avec une fréquence significativement augmentée chez les enfants nés de mères traitées par Valproate de sodium [28]. Des études plus anciennes ont également associé la prise de barbituriques pendant la grossesse à un moins bon développement cognitif [29]. Enfin, la polythérapie est également associée dans plusieurs études à un moindre devenir cognitif.

Concernant, le devenir cognitif des enfants nés de mères traitées par Carbamazépine, Lamotrigine, Topiramate ou Lévétiracétam [30,31], les données, quoi que rassurantes, demeurent parcellaires, sur de faibles effectifs avec souvent le biais du Valproate de Sodium pris comme comparateur. Des études prospectives de plus grande ampleur sont requises. Pour les autres MAE, on ne dispose à ce jour d'aucune donnée.

\section{Troubles autistiques}

En 2013, une large étude de cohorte danoise a retrouvé un risque accru d'autisme infantile ( $2.5 \%$ vs $0.5 \%$ dans la population contrôle) et de troubles du spectre autistique $(4.4 \%$ vs $1.5 \%$ ) chez des enfants nés de mères ayant pris du Valproate pendant leur grossesse [32].

\section{Surveillance de la grossesse}


Les données issues d'EURAP montrent que le risque de crises est identique tout au long de la grossesse et que le fait d'avoir une épilepsie bien équilibrée dans les mois précédant la grossesse est un facteur prédictif de bon équilibre tout au long de la grossesse. Une autre étude récente issue d'EURAP montre que l'arrêt ou le remplacement du Valproate de sodium en cours de grossesse est associé à une augmentation du risque de survenue de crise généralisée tonico-clonique [33]. La survenue de crises, notamment de crises généralisées tonico-cloniques, pendant la grossesse expose au risque [34,35]: 1) de chute avec potentiel traumatisme obstétrical, 2) de prématurité, 3) d'hypotrophie fœetale et 4) de mort fœetale en cas de survenue d'un état de mal tonico-clonique généralisé.

Pendant la grossesse, une prise en charge coordonnée multidisciplinaire (neurologueobstétricien-anesthésiste-pédiatre) est requise. La modification des facteurs pharmacocinétiques imposera des dosages plasmatiques itératifs et réguliers des médicaments anti-épileptiques (avec si possible un référentiel pré grossesse) pour un éventuel ajustement des doses (parfois à titre préventif). Un suivi échographique de bonne qualité est requis en indiquant bien que la patiente prend des traitements anti-épileptiques et lesquels. Un dépistage de la dépression du post-partum est fortement recommandé chez la patiente épileptique.

\section{Accouchement et allaitement}

Bortehn et al.[36] ont montré que les femmes épileptiques avaient un faible taux de complications à l'accouchement mais néanmoins un risque augmenté d'accouchement déclenché, ou par césarienne, témoignant certainement de la crainte des obstétriciens de la survenue d'une crise au moment de l'accouchement. Néanmoins, le risque de crises se semble absolument pas augmenté lors de l'accouchement (3.5\% dans EURAP). Un accouchement naturel par voie basse, lorsque les conditions obstétricales le permettent, est donc tout à fait conseillé et la péridurale chaudement recommandée. Le traitement anti-épileptique habituel doit être poursuivi pendant le travail et l'accouchement. Dans tous les cas, le nouveau-né recevra une injection de vitamine $\mathrm{K} 1$ à dose standard (2 mg per os ou $\mathrm{IM}$ ) ou une dose à risque hémorragique majoré ( 0,5 à $1 \mathrm{mg} \mathrm{IM}$ ou IV lente de vitamine $\mathrm{K} 1)$ si la mère recevait des inducteurs enzymatiques et notamment du phénobarbital.

Concernant l'allaitement, il existe actuellement une controverse sur le fait d'autoriser ou non les femmes épileptiques à allaiter avec parfois des visions différentes de la part des neurologues, des obstétriciens et des pédiatres. Différents paramètres vont entrer en jeu : 1)le degré d'excrétion du médicament dans le lait maternel (faible, moyen ou fort) (tableau 2), 2) le terme de l'accouchement : l'immaturité du système enzymatique du nouveau-né qui favorise l'accumulation des médicaments est encore plus accentuée en cas de prématurité, et 
3) la durée de l'allaitement : plus l'allaitement est pratiqué sur une période prolongée , plus le risque d'accumulation des MAE et donc d'effets secondaires augmente chez le nouveau né. Une étude récente [37] a montré que l'allaitement avait un effet bénéfique à long terme (suivi à 6 ans) sur les enfants allaités nés de mères épileptiques traitées par différents MAE (carbamazépine, lamotrigine, phénytoïne et valproate de sodium) versus des enfants non allaités nés de mères prenant les mêmes MAE. En pratique, la décision d'allaitement demeure individuelle, prise conjointement en accord avec : la patiente, le neurologue, le gynécologue et le pédiatre. Cette décision sera fonction : 1) du désir de la patiente d'allaiter ; 2) du terme de l'accouchement (déconseillé chez les prématurés), 3) du traitement anti-épileptique (nombre et type de MAE, allaitement déconseillé en cas de polythérapie ou de médicaments très sédatifs (benzodiazèpines notamment, barbituriques), à considérer au cas par cas en cas de monothérapie).

\section{En pratique}

Chaque prise en charge demeurera individuelle, fonction du type d'épilepsie et du traitement en place, mais de grands principes pourront être respectés :

- proscrire le valproate de sodium en cas de grossesse : le remplacer idéalement avant la grossesse ou sinon l'arrêter en tout début de grossesse (quitte à le reprendre dès

l'accouchement en cas de déséquilibre grave de l'épilepsie)

- privilégier une monothérapie à base de Lamotrigine ou Lévétiracétam

- en cas de monothérapie par Carbamazépine : on peut envisager la grossesse en essayant de réduire les doses en-dessous de $800 \mathrm{mg} / \mathrm{j}$

- fractionner au maximum les prises sur la journée

- prendre un avis spécialisé au moindre doute (polythérapie, médicaments pour lesquels on n'a pas de recul, épilepsie très active.....)

\section{Ménopause}

La ménopause est définie comme l'arrêt définitif des menstruations en rapport avec la perte de la fonction ovarienne folliculaire. Elle survient aux alentours de 51 ans dans la population générale (42-58 ans) mais a tendance à être plus précoce de 2 à 3 ans chez les patientes épileptiques [38]. Il existe relativement peu d'études de la littérature sur l'effet de la ménopause sur l'équilibre des crises. La plupart des études ne retrouvent pas d'effet probant sur la fréquence des crises [39], exception faite des patientes avec épilepsie cataméniale qui 
peuvent noter une exacerbation de leurs crises en période de péri ménopause puis une amélioration une fois la ménopause avérée [40]. Une des préoccupations majeures chez la patiente épileptique ménopausée est l'accroissement du risque d'ostéoporose lié tout à la fois à l'âge et aux modifications hormonales mais aussi à la prise au long cours des MAE qui ont per se un effet délétère sur la minéralité osseuse [41]. Cet effet délétère connu de longue date avec les MAE inducteurs enzymatiques [42] semble également exister avec les nouvelles générations non inductrices de MAE [43]. Chez les patientes épileptiques ménopausées traitées au long cours par MAE, on recommande la réalisation d'une ostéodensitométrie pour étudier la densité osseuse et le cas échéant des traitements substitutifs par calcium et vitamine D voire biphosphonates [44]. Concernant l'effet de l'hormonothérapie substitutive de la ménopause sur l'équilibre des crises, les données restent parcellaires, une étude randomisée a démontré un effet aggravant sur la fréquence des crises d'une thérapie associant des estrogènes équins conjugués et de l'acétate de medroxyprogestérone [45] mais ce type d'hormonothérapie n'est pas employé en France.

\section{Conclusion}

La prise en charge de l'épilepsie chez la femme nécessite non seulement une connaissance de la maladie mais aussi la reconnaissance des rôles variés et spécifiques des femmes au cours de leur vie (sexualité, contrôle des naissances, grossesses, éducation des enfants, carrière professionnelle....). Bien prendre en charge une patiente épileptique, c'est prêter avant tout une attention soutenue et une réponse à leurs besoins et demandes spécifiques. 


\section{Références}

[1] Herzog AG, Klein P, Ransil BJ. Three patterns of catamenial epilepsy. Epilepsia 1997;38:1082-8.

[2] Herzog AG. Catamenial epilepsy: definition, prevalence, pathophysiology and treatment. Seizure 2008;17(2):151-9.

[3] Herzog AG. Catamenial epilepsy: Update on prevalence, pathophysiology and treatment from the findings of the NIH Progesterone Treatment Trial. Seizure 2015;28:18-25.

[4] A.G. Herzog, K.M. Fowler, S.D. Smithson, L.A. Kalayjian, C.N. Heck, M.R. Sperling, et al., the Progesterone Trial Study Group Progesterone versus placebo therapy for women with epilepsy: a randomized clinical trial Neurology 2012;78:1959-1966

[5] Pack AM, Davis AR, Kritzer J, Yoon A, Camus A. Antiepileptic drugs: arewomen aware of interactions with oral contraceptives and potential teratogenicity? Epilepsy Behav

2009;14:640-644.

[6] Fairgrieve SD, Jackson M, Jonas P, et al. Population based, prospective study of the care of women with epilepsy in pregnancy. BMJ 2000;321:674-675.

[7] Centers for Disease Control and Prevention (CDC). U.S. medical eligibility criteria for contraceptive use, 2010. MMWR Recomm Rep 2010;59:1-86.

[8] Gaffield ME, Culwell KR, Lee CR. The use of hormonal contraception among women taking anticonvulsant therapy. Contraception 2011;83:16-29.

[9] Reddy DS Clinical pharmacokinetic interactions between antiepileptic drugs and hormonal contraceptives. Expert Rev Clin Pharmacol. 2010;3(2):183-192.

[10] Sabers A, Ohman I, Christensen J, Tomson T. Oral contraceptives reduce lamotrigine plasma levels. Neurology 2003;61:570-571.

[11] Galimberti CA, Mazzucchelli I, Arbasino C, Canevini MP, Fattore C, Pericca E. Increased apparent oral clearance of valproic acid during intake of combined contraceptive steroids in women with epilepsy. Epilepsia 2006;47:1569-72.

[12] Herzog AG, Mandle HB, Cahill KE, Fowler KM, Hauser WA Differential impact of contraceptive methods on seizures varies by antiepileptic drug category: Findings of the Epilepsy Birth Control Registry Epilepsy \& Behavior 2016;60:112-117

[13] Appendix C: AAN Summary of Evidence-Based Guideline for Clinicians: Management Issues for Women With Epilepsy--Focus on Pregnancy: Vitamin K, Folic Acid, Blood Levels, and Breastfeeding. Continuum (Minneap Minn). 2016;22:285-6.

[14] Hernández-Díaz S, Smith CR, Shen A, Mittendorf R, Hauser WA, Yerby M, Holmes LB; North American AED Pregnancy Registry; North American AED Pregnancy Registry Comparative safety of antiepileptic drugs during pregnancy Neurology. 2012 ;78(21):16921699

[15] Morrow J, Russell A, Guthrie E, Parsons L, Robertson I, Waddell R, Irwin B, McGivern RC, Morrison PJ, Craig J Malformation risks of antiepileptic drugs in pregnancy: a prospective study from the UK Epilepsy andPregnancy Register J Neurol Neurosurg Psychiatry. 2006;77(2):193-198.

[16] Campbell E, Kennedy F, Russell A, et al. Malformation risks of antiepileptic drug monotherapies in pregnancy: updated results from the UK and Ireland Epilepsy and Pregnancy Registers. J Neurol Neurosurg Psychiatry 2014;85:1029-1034.

[17] L. Holmes, J. A. French, W. A. Hauser, P. G. Wells, J. A. Cramer and For the HOPE K. J. Meador, P. B. Pennell, C. L. Harden, J. C. Gordon, T. Tomson, P. W. Kaplan, G. Tomson T, Battino D, Bonizzoni E, JCraig J, Lindhout D, Sabers A, Perucca E, Vajda F, for the EURAP 
study group Dose-dependent risk of malformations with antiepileptic drugs: an analysis of data from the EURAP epilepsy and pregnancy registry Lancet Neurol 2011;10: 609-17 [18] Weston J, Bromley R, Jackson CF, Adab N, Clayton-Smith J, Greenhalgh J, Hounsome J, McKay AJ, Tudur Smith C, Marson AG Monotherapy treatment of epilepsy in pregnancy: congenital malformation outcomes in the child (Review) Cochrane Database of Systematic Reviews 2016;11. Art. No.: CD010224.

[19] Veroniki AA, Cogo E, Rios P, Straus SE, Finkelstein Y, Kealey R, Reynen E, Soobiah C, Thavorn K, Hutton B, Hemmelgarn BR, Yazdi F, D'Souza J, MacDonald H, Tricco AC Comparative safety of anti-epileptic drugs during pregnancy: a systematic review and network meta-analysis of congenital malformations and prenatal outcomes BMC Med. 2017;15(1):95 [20] Margulis AV, Mitchell AA, Gilboa SM, et al; National Birth Defects Prevention Study. Use of topiramate in pregnancy and risk of oral clefts. Am J Obstet Gynecol 2012; 207: 405: e1-7.

[21] Dolk H, Wang H, Loane M, Morris J, Garne E, Addor MC, Arriola L, Bakker M, Barisic I, Doray B, Gatt M, Kallen K, Khoshnood B, Klungsoyr K, Lahesmaa-Korpinen AM, LatosBielenska A, Mejnartowicz JP, Nelen V, Neville A, O'Mahony M, Pierini A, Rißmann A, Tucker D, Wellesley D, Wiesel A, de Jong-van den Berg LT. Lamotrigine use in pregnancy and risk of orofacial cleft and other congenital anomalies. Neurology. 2016;86(18):1716-1725. [22] Montouris G. Safety of the newer antiepileptic drug oxcarbazepine during pregnancy Curr Med Res Opin. 2005;21(5):693-701.

[23] Winterfeld U, Merlob P, Baud D, Rousson V, Panchaud A, Rothuizen LE, Bernard N, Vial T, Yates LM, Pistelli A, Ellfolk M, Eleftheriou G, de Vries LC, Jonville-Bera AP, Kadioglu M, Biollaz J, Buclin T Pregnancy outcome following maternal exposure to pregabalin may call for concern Neurology. 2016;86(24):2251-2257.

[24] Vajda FJ, O'Brien TJ, Lander CM, Graham J, Eadie MJ. Antiepileptic drug combinations not involving valproate and the risk of fetal malformations Epilepsia. 2016 ;57(7):1048-52. [25] Adab N, Kini U, Vinten J, Ayres J, Baker G, Clayton-Smith J, et al. The longer term outcome of children born to mothers with epilepsy. J Neurol Neurosurg Psychiatry 2004; 75:1575-1583.

[26] Meador K, Baker G, Browning N, Clayton-Smith J, Combs-Cantrell D, Cohen M, et al. 2009. Cognitive function at 3 years of age after fetal exposure to antiepileptic drugs. N Engl J Med; 360:1597-1605.

[27] Nadebaum C, Anderson V, Vajda F, Reutens D, Barton S, Wood A. 2011. Language skills of school-aged children prenatally exposed to antiepileptic drugs. Neurology; 76:719726.

[28] Deshmukh U, Adams J, Macklin EA, Dhillon R, McCarthy KD, Dworetzky B, Klein A, Holmes LB. Behavioral outcomes in children exposed prenatally to lamotrigine, valproate, or carbamazepine Neurotoxicol Teratol. 2016;54:5-14.

[29] Koch S, Titze K, Zimmermann RB, Schröder M, Lehmkuhl U, Rauh H. Long-term neuropsychological consequences of maternal epilepsy and anticonvulsant treatment during pregnancy for school-age children and adolescents. Epilepsia. 1999;40(9):1237-43.

[30] Bromley R, Weston J, Adab N, Greenhalgh J, Sanniti A, McKay AJ, Tudur Smith C, Marson AG Treatment for epilepsy in pregnancy: neurodevelopmental outcomes in the child. Cochrane Database Syst Rev. 2014;10:CD010236.

[31] Bromley RL, Calderbank R, Cheyne CP, Rooney C, Trayner P, Clayton-Smith J, GarcíaFiñana M, Irwin B, Morrow JI, Shallcross R, Baker GA; UK Epilepsy and Pregnancy Register. Cognition in school-age children exposed to levetiracetam, topiramate, or sodium valproate. Neurology. 2016;87(18):1943-1953. 
[32]Christensen J, Koops Grønborg T, Sørensen MJ, Schendel D, Parner ET, Pedersen LH, Vestergaard M Prenatal Valproate Exposure and Risk of Autism Spectrum Disorders and Childhood Autism JAMA 2013; 309:16

[33 ]Group ES. Seizure control and treatment in pregnancy: observations from the EURAP epilepsy pregnancy registry. Neurology 2006; 66:354-360.

[34] Rauchenzauner M, Ehrensberger M, Prieschl M, Kapelari K, Bergmann M, Walser G, Neururer S, Unterberger I, Luef G. Generalized tonic-clonic seizures and antiepileptic drugs during pregnancy--a matter of importance for the baby? J Neurol. 2013;260(2):484-488.

[35] Chen D, Hou L, Duan X, Peng H, Peng B. Effect of epilepsy in pregnancy on fetal growth restriction: a systematic review and meta-analysis. Arch Gynecol Obstet. 2017 [36] Borthen I, Eide MG, Daltveit AK, Gilhus NE. Delivery outcome of women with epilepsy: a population-based cohort study. BJOG. 2010 ;117(12):1537-1543.

[37 ] Meador KJ, Baker GA, Browning N, Cohen MJ, Bromley RL, Clayton-Smith J, Kalayjian LA, Kanner A, Liporace JD, Pennell PB, Privitera M,Loring

DW Neurodevelopmental Effects of Antiepileptic Drugs (NEAD) Study Group Breastfeeding in children of women taking antiepileptic drugs: cognitive outcomes at age 6 years JAMA Pediatr. 2014 ;168(8):729-36.

[38] Klein P, Serje A, Pezzulo JC. Premature ovarian failure in women with epilepsy. Epilepsia. 2001;42:1584-9.

[39] Abbasi F, Krumholz A, Kittner SJ, et al. Effects of menopause on seizures in women with epilepsy. Epilepsia. 1999;40:205-10.

[40] Harden CL, Pulver MC, Ravdin L, et al. The effect of menopause and perimenopause on the course of epilepsy. Epilepsia 1999;40:1402-1407.

[41] McAuley JW, Sapna SJ, Moore JL, et al. Characterization and health risk assessment of postmenopausal women with epilepsy.Epilepsy Behav. 2000;1(353-355):23.

[42] Petty SJ, Paton LM, O_Brien TJ et al. Effect of antiepileptic medication on bone mineral measures. Neurology 2005;65:1358-65.

[43] Beerhorst K, Schouwenaars FM, Tan IY, Aldenkamp AP. Epilepsy: fractures and the role of cumulative antiepileptic drug load. Acta Neurol Scand. 2012;125(1):54-59.

[44] Erel CT, Brincat M, Gambacciani M, Lambrinoudaki I, Moen MH, Schenck-Gustafsson $\mathrm{K}$, Tremollieres F, Vujovic S, Rozenberg S, Rees M. EMAS position statement: managing the menopause in women with epilepsy. Maturitas. 2010 ;66(3):327-328.

[45] Harden CL, Herzog AG, Nikolov BG, et al. Hormone replacement therapy in women with epilepsy: a randomized, double-blind, placebo-controlled study. Epilepsia2006;47:144751 . 
Légendes tableaux et figures

Tableau 1 : MAE et contraception

Tableau 2 : Excrétion des MAE dans le lait maternel

Figure 1 : Formulaire d'accord de soins Valproate 